\title{
Efecto de la saturación de agua durante la ignición de un proceso de combustión in situ
}

\section{Effect of water saturation during the ignition of an in situ combustion process}

\section{Efeito da saturação de agua durante a ignição de um processo de combustão in-situ}

\author{
Juan Dangon Molano ${ }^{1 *}$; Silvia Mora Uribe ${ }^{1}$; Samuel Muñoz N. ${ }^{1}$; Jorge Padilla R. ${ }^{1}$; Hernando Bottía \\ Ramirez ${ }^{2}$ \\ ${ }^{1}$ Grupo de Investigación Recobro Mejorado. Universidad Industrial de Santander (UIS), Bucaramanga, Colombia \\ 2Instituto Colombiano del Petróleo, ECOPETROL-ICP, Piedecuesta, Colombia \\ *juan.molano1@correo.uis.edu.co
}

Fecha recepción: 11 de octubre de 2017 Fecha aceptación: 10 de septiembre de 2018

\begin{abstract}
Resumen
Combustión In Situ (CIS) es un método térmico para la recuperación mejorada de crudo pesado. Para iniciar el proceso de CIS es necesario consumir una parte del aceite en la formación mediante reacciones de oxidación y luego mantener la combustión mediante la inyección continua de aire. Esta etapa inicial es conocida como la ignición del proceso y su estudio permite identificar el requerimiento energético del sistema roca-fluido. La correcta investigación de la ignición puede conducir a una aplicación exitosa en campo de CIS.

El propósito del presente trabajo es predecir la ignición espontánea de un yacimiento de crudo pesado colombiano mediante un modelo matemático programado en MATLAB, el cual tiene en cuenta la transferencia de masa y energía que ocurre en el régimen de oxidación a bajas temperaturas (LTO). Los parámetros cinéticos fueron obtenidos a partir de las pruebas de celda isotérmica realizadas en el laboratorio de inyección de aire del Instituto Colombiano del Petróleo. Los resultados mostraron el efecto directo de la saturación de agua sobre el tiempo de retardo y la distancia a la cual toma lugar la ignición espontánea desde el pozo inyector.
\end{abstract}

Palabras clave: Combustión in situ, prueba de celda isotérmica, ignición espontánea, MATLAB.

\begin{abstract}
In Situ Combustion (ISC) is a thermal method for the enhanced heavy oil recovery. To start the ISC process is necessary to consume a part of the oil in the reservoir by means of the oxidation reactions, then maintain combustion by continuous air injection. This initial stage is known as ignition and its study allows to identify the energy requirement of the rock-fluid system. Proper research of ignition could lead to successful ISC field trial.

The purpose of the present project is to predict the spontaneous ignition of a Colombian heavy oil reservoir through a mathematical model programmed in MATLAB, which took into account a combined mass and heat transfer of the reactions that occur in the low temperature oxidation (LTO) regime. The kinetic parameters were obtained from the isothermal cell tests performed in the Air Injection Laboratory
\end{abstract}

Cita: Dangon Molano J, Mora Uribe S, Muñoz S, Padilla J, Bottia Ramúrez H. Efecto de la saturación de agua durante la ignición de un proceso de combustión in situ. rev.ion. 2018;31(2):83-97. doi:10.18273/revion.v31n2-2018006 
of the Instituto Colombiano del Petróleo. The results showed the direct effect of the water saturation on the delay ignition and the ignition distance from the injector well.

Keywords: In Situ Combustion, Isothermal Cell Unit, Spontaneous Ignition, MATLAB.

\section{Resumo}

Combustão in-situ (CIS) é um método de recuperação térmica melhorada óleo pesado. Para iniciar o processo CIS é necessário consumir uma parte do óleo na formação por meio de reações de oxidação e, em seguida, manter a combustão mediante a injeção continua de ar. Esta fase inicial e conhecida como a ignição do processo e seu estudo identifica a exigência de energia o sistema rock fluido. A pesquisa correta da ignição pode levar a uma aplicação o CIS bem-sucedida no campo.

O objetivo deste trabalho é prever a ignição espontânea de um reservatório de óleo pesado colombiano mediante um modelo matemático programado em MATLAB que leva em conta a transferência de massa e energia que ocorre no regime de oxidação a temperatura baixa (LTO). Os parâmetros cinéticos foram obtidos a partir de ensaios realizados no Laboratório de injeção de ar do Instituto Colombiano de Petróleo. Os resultados mostraram o efeito direito da saturação de agua sobre o tempo de atraso e a distância onde ocorre a ignição espontânea desde o poço injetor.

Palavras-chave: combustão in-situ, prova de celular isotérmica, Ignição Espontâneo, MATLAB.

\section{Introducción}

Colombia cuenta con más de un $38 \%$ de reservas que corresponden a crudo pesado y extra pesado, en yacimientos de este tipo el factor de recobro es solo del $6 \%$. La recuperación de crudo pesado es compleja debido a que este tipo de fluido se caracteriza por tener alta viscosidad, alta densidad y bajas propiedades de fluidez [1]. Por lo tanto, para su extracción es necesario emplear métodos de recuperación mejorada de petróleo que atenúen estas condiciones adversas de movilidad.

Uno de estos métodos es la recuperación térmica, que reduce la viscosidad en varios órdenes de magnitud mediante el incremento de la temperatura [2]. Dentro de esta modalidad se encuentra la Combustión In Situ (CIS), considerada como una de las técnicas más eficientes para la recuperación mejorada de petróleo. Esta técnica permite la liberación de energía dentro del yacimiento a partir de reacciones exotérmicas entre el hidrocarburo y el oxígeno inyectado.

Al momento de planear un proceso de recobro mediante CIS, uno de los principales problemas es el inicio de la propagación estable del frente de combustión, denominada ignición. Esta se produce cuando la reacción entre el crudo presente en el yacimiento y el oxígeno contenido en el gas inyectado alcanzan la energía necesaria para dar lugar a las reacciones de oxidación total [3]. El requerimiento energético, usualmente es identificado a partir de la temperatura de ignición, esta puede ser alcanzada mediante fuentes externas de calor, ignición asistida, o el calor liberado por las reacciones a bajas temperaturas (LTO), ignición espontánea. En cualquier proceso de CIS, la ignición juega un papel esencial, no solo para iniciar el frente de combustión sino también para mantener un control adecuado de un proceso de CIS y garantizar la propagación del frente de combustión [4].

De acuerdo con Alex Turta [5], la ignición espontánea se encuentra dominada por las reacciones LTO y sólo se presentará en yacimientos con temperaturas mayores de 60-70 ${ }^{\circ} \mathrm{C}$. El tiempo que tarda en generarse el frente de combustión, conocido como tiempo de retardo, depende de la temperatura inicial de la formación y la reactividad del crudo, puede variar entre unas semanas a meses.

Burger y Sahuquet [6] mostraron que los mayores productos generados en las reacciones LTO son hidrocarburos oxigenados, tales como alcoholes, aldehídos, hidroperóxidos, cetonas y ácidos carboxílicos. El entendimiento de las reacciones LTO es de gran importancia, ya que sus productos juegan un rol importante en la etapa estable de un proceso de CIS. Por ejemplo, la producción excesiva de los productos de las reacciones LTO puede ocasionar bloqueo del medio poroso, debido a que la viscosidad de la mezcla de hidrocarburos que contiene estos productos es mayor que la del crudo original [7].

Donald Lee y Nazih Noureldin [8] demostraron 
cómo la presencia de agua disminuye el efecto destructivo de las reacciones LTO, haciendo que los productos de estas reacciones parciales tengan menor acidez y menor viscosidad. Hascakir y Klock [9], evaluaron el efecto de la heterogeneidad de las saturaciones de los fluidos sobre las reacciones químicas que se desarrollan en un proceso de CIS, encontrando que el agua es un componente favorable para la sostenibilidad del proceso de combustión en sistemas con altas saturaciones de aceite y altas tasas de calentamiento.

Para el presente trabajo de investigación se consideró la construcción de una herramienta computacional que fuera capaz de representar el comportamiento de la ignición espontánea en un yacimiento de crudo pesado. El modelo matemático fue seleccionado con base en los estudios reportados por diferentes autores, quienes modelaron los diferentes mecanismos de transferencia de masa y energía que gobiernan la ignición espontánea de un proceso de CIS.

En 1968 Roger Schoppel y Denir Ersoy [10], presentaron un estudio teórico de la ignición espontánea como una etapa intencional o no intencional en el proceso de CIS. Este estudio se realizó con base en un análisis de la transferencia de calor y de masa de las reacciones de oxidación a bajas temperaturas. Ellos lograron predecir la ignición espontánea en un pozo sin necesidad de fuentes externas de calor. Además, evaluó el efecto de las variables que pueden ser controladas durante el proceso como la temperatura inicial, la actividad química del crudo, la cantidad de oxigeno presente en el aire y la presión.

En 1970 H.J. Tadema y J. Weijdema [11], propusieron una expresión analítica para tener una estimación del tiempo de ignición en función de las tasas de oxidación del crudo. Los autores consideraban que el tiempo de la ignición espontánea dependía de la temperatura inicial de la formación y de la reactividad del crudo, por lo que el tiempo de ignición podía variar desde días a meses. Además, esperaban que la ignición iniciara en una zona a cierta distancia del pozo de inyección debido a la perdida de calor a lo largo del revestimiento. En esta ecuación se asumió que el efecto de transferencia de calor por conducción y por convección del gas era insignificante.

En 1976, Jacques Burger [12] planteó un modelo numérico para representar las condiciones de ignición y determinar la posición de la zona de ignición en función de las características del yacimiento, del aceite y del flujo de gas. En su modelo, el flujo de gas se asumió unidimensional y se consideraron los efectos de transferencia de calor por conducción y convección durante la ignición. Para Burger, la presión y la tasa de flujo de gas eran constantes alrededor del pozo y en el balance de calor no se tenía en cuenta la vaporización del agua ni la difusión del oxígeno. Con este modelo evaluó el tiempo de ignición, el calentamiento exponencial del proceso y el efecto de la tasa de inyección de aire para un crudo pesado.

En 1985 C. Agca y Y.C. Yortsos [13] presentaron una alternativa para simplificar el análisis del estado estable del proceso de CIS. Mediante ecuaciones de conservación de masa y energía térmica lograron representar el proceso de combustión dinámica. Los autores consideraron que para una ignición espontánea resulta favorable contar con altas temperaturas iniciales de yacimiento, altas tasas y presiones de inyección, así como también reducir las pérdidas de calor.

El modelo matemático seleccionado para el presente trabajo de investigación fue el de Roger Schoppel y Denir Ersoy [10], ya que tiene en cuenta un número considerable de mecanismos de trasferencia de masa y energía, y permite obtener distribuciones de temperatura y de concentraciones de oxígeno a través del yacimiento.

\section{Metodología}

La herramienta programada tiene como propósito representar gráficamente los cambios de temperatura y de las concentraciones de oxígeno durante el proceso de oxidación de un crudo colombiano. Para poder obtener las gráficas anteriormente mencionadas, la herramienta requiere parámetros específicos de entrada, siendo la cinética de reacción una de las más importantes.

Con el fin de determinar la cinética de reacción del crudo a estudiar, el Instituto Colombiano del Petróleo (ICP) realizó pruebas de celda isotérmica, cuyos resultados fueron utilizados para el desarrollo del presente trabajo.

\section{Celda de oxidación isotérmica}

La celda de oxidación isotérmica del ICP es una unidad utilizada para medir la cinética de oxidación del crudo en la matriz del yacimiento [3]. El proceso consiste en determinar la velocidad de oxidación del aceite a diferentes temperaturas y diferentes presiones, a partir del cambio en el contenido de 
oxígeno introducido en la celda al inicio del ensayo, a una temperatura constante dada [14].

La primera hipótesis en el desarrollo de estas pruebas es considerar que el crudo es equivalente a un solo tipo de hidrocarburo y no a una mezcla de varios tipos, la segunda es considerar que solo hay una reacción de oxidación y no existen reacciones consecutivas o en paralelo. El esquema de reacción de adición (Ecuación 1) utilizado para representar las reacciones de oxidación a bajas temperaturas (LTO) en los experimentos de celda isotérmica fue el propuesto por Burger [12]:

$$
\text { Heavy Oil }+ \text { Oxygen } \rightarrow \mathrm{CO}+\mathrm{CO}_{2}+\mathrm{HCOH}+\mathrm{HCOOH}+\text { water }
$$

\section{Resultados}

A continuación, se presentan los resultados obtenidos de las pruebas de celda isotérmica para el crudo estudiado con el fin de determinar los parámetros cinéticos y para evaluar el efecto que tiene el agua sobre los mismos. Los datos recolectados de estas pruebas fueron otorgados por el personal del laboratorio de inyección de aire del Instituto Colombiano del Petróleo. Adicionalmente, se presenta el planteamiento, programación y validación del modelo matemático empleado para evaluar el efecto de la saturación de agua durante la ignición espontánea del crudo estudiado.

Determinación de la cinética de oxidación para un sistema aceite-roca

EI ICP desarrolló ocho pruebas de celda isotérmica, las cuales consistieron en reproducir las condiciones de oxidación del crudo a condiciones de $8,27 \mathrm{MPa}$ (1200 psi) y $26,20 \mathrm{MPa}$ (3800 psi). Las condiciones a las cuales se realizaron las pruebas experimentales se encuentran en la Tabla 1. Para realizar el cálculo de la cantidad de oxígeno en la etapa final del ensayo se hace uso del analizador de gases que se encuentra a la salida del reactor. Una vez finalizada la prueba se calcula la fracción molar $X_{O 2}$ que corresponde al oxígeno a las condiciones de temperatura y presión de ensayo (Ecuación 2). El cual es calculado usando la información dada por el analizador de gases, el gasómetro y las moles de $\mathrm{O}_{2}$ iniciales introducidas al sistema con el aire.

$$
X_{\mathrm{O}_{2}}=\frac{\text { moles } \mathrm{O}_{2}}{\text { moles totales }}
$$

Por último, se puede proceder a calcular la velocidad especifica de reacción $K$ (Ecuación 3 ). (Ver Tablas 2 y 3 ).

$$
K=\frac{p^{*} v^{*}\left[O_{2 i}-O_{2 f}\right]}{t} * \frac{1}{z R T} * \frac{M_{O_{2}}}{M_{O}}
$$

Tabla 1. Parámetros de operación para las pruebas celda de oxidación isotérmica.

\begin{tabular}{ccc}
\hline Condición de la prueba & $\begin{array}{c}\text { Celda } \\
\text { Gas inyectado }\end{array}$ & $\begin{array}{c}\text { Celda } \\
\mathbf{2 6 , 2 0} \mathbf{~ M P a ~ ( 3 8 0 0 ~} \mathbf{~ p s i})\end{array}$ \\
\hline Temperaturas de los ensayos $\left({ }^{\circ} \mathrm{C}\right)$ & Aire & Aire \\
Presión de Operación (MPa) & $100 ; 120 ; 140$ & $109 ; 125 ; 130 ; 150 ; 153$ \\
Tiempo de la prueba (Horas en promedio) & 8,27 & 26,20 \\
Concentración de oxigeno, $\%$ mol & 24 & 63,$91 ; 25,80 ; 22,66 ; 18,85 ; 20,33$ \\
\hline
\end{tabular}


Tabla 2. Velocidades de reacción calculadas para el sistema aceite-roca a presión de 8,27 MPa (1200 psi).

\begin{tabular}{|c|c|}
\hline $\begin{array}{l}\text { Temperatura Promedio } \\
{\left[{ }^{\circ} \mathrm{C}\right]}\end{array}$ & $\mathrm{K}\left[\mathrm{g} \mathrm{O}_{2} / \mathrm{g}\right.$ oil*s] \\
\hline 99,77 & 1,11 E-06 \\
\hline 117,37 & 2,47 E-06 \\
\hline 137,79 & 4,53 E-06 \\
\hline
\end{tabular}

Tabla 3. Velocidades de reacción calculadas para el sistema aceite-roca a presión de 26,20 MPa (3800 psi).

\begin{tabular}{ccc}
\hline $\begin{array}{c}\text { Temperatura } \\
\text { Promedio }\left[{ }^{\circ} \mathbf{C}\right]\end{array}$ & Tiempo $[\mathbf{s}]$ & $\boldsymbol{K}$ [g O $\left.\mathbf{~}_{\mathbf{2}} \mathbf{\text { g oil }}{ }^{*} \mathbf{s}\right]$ \\
\hline 109,21 & 230100 & $1,87 \mathrm{E}-06$ \\
125,87 & 92880 & $6,05 \mathrm{E}-06$ \\
130,08 & 81600 & $7,53 \mathrm{E}-06$ \\
149,59 & 67860 & $1,58 \mathrm{E}-05$ \\
153,45 & 73200 & $2,02 \mathrm{E}-05$ \\
\hline
\end{tabular}

Teniendo en cuenta la ecuación de Arrhenius (Ecuación 4), se aplica una linealización, luego una regresión lineal usando mínimos cuadrados y finalmente se calculan los parámetros cinéticos con los mejores valores que representarán la cinética a bajas temperaturas.

$$
K=k_{0} e^{\frac{-E}{R T}} p_{O_{2}}^{n}
$$

Linealizando se obtiene la expresión (Ecuación 5),

$$
\operatorname{Ln} K=\operatorname{Ln}\left[k_{0} p_{O_{2}}^{n}\right]-E / R T
$$

Con los valores de velocidad de reacción calculadas a diferentes temperaturas se construye la gráfica $K$ vs $1 / T$ (Figura 1).

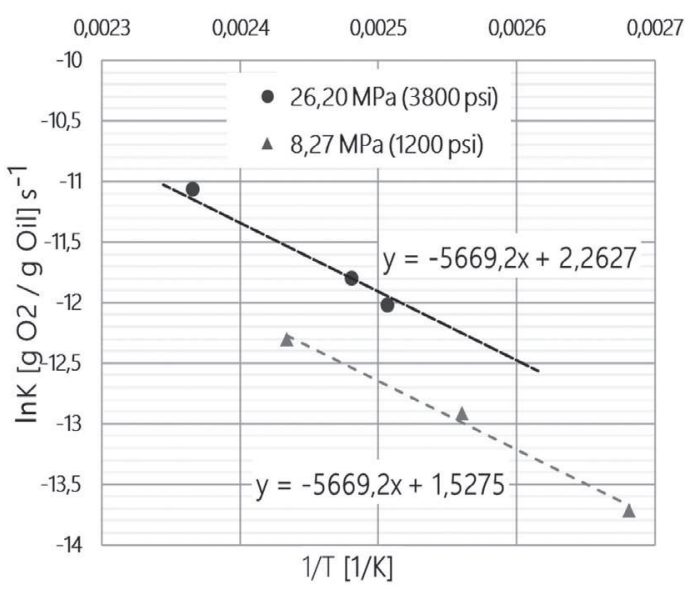

Figura 1. Gráfica de K vs 1/T para un sistema aceite-roca.

De la Figura 1 se obtienen los parámetros cinéticos: factor pre-exponencial $k_{o}$, la energía de activación $E$ y el orden de reacción $n$ (Tabla 4).

Tabla 4. Parámetros cinéticos del sistema aceite - roca.

\begin{tabular}{ccc}
\hline Celda & Parámetro & Valor \\
\hline $\mathbf{8 , 2 7} \mathbf{~ M P a}$ & $E / R[\mathrm{~K}]$ & $-5569,20$ \\
$(\mathbf{1 2 0 0} \mathbf{~ p s i )}$ & $k_{01} p_{10_{2}}^{n}\left[\mathrm{~atm}^{\mathrm{n}} / \mathrm{s}\right]$ & 4,61 \\
\hline $\mathbf{2 6 , 2 0} \mathbf{~ M P a}$ & $E / R[\mathrm{~K}]$ & $-5569,20$ \\
$\mathbf{( 3 8 0 0} \mathbf{~ p s i )}$ & $k_{01} p_{10_{2}}^{n}\left[\mathrm{~atm}^{\mathrm{n}} / \mathrm{s}\right]$ & 9,61 \\
\hline
\end{tabular}

Como se trata del mismo sistema aceite-roca se considera que tanto $k_{o}$ como $n$ son iguales, entonces la cinética completa es la siguiente (Ecuación 6):

$$
K=0,778 \cdot p_{O_{2}}^{0,63} e^{\frac{-5569,2}{T}}
$$

Efecto de la saturación de agua sobre la cinética de oxidación

Con el fin de evaluar el efecto de la saturación de agua inicial sobre la cinética de oxidación del aceite en presencia de matriz de afloramiento y agua, el ICP realizó pruebas adicionales de celda isotérmica con muestras de solo crudo y crudo agua a 100,120 y $140^{\circ} \mathrm{C}$, todas fueron realizadas a la presión actual del yacimiento, 8,27 MPa (1200 psi) (Tabla 5). La cinética de reacción para los dos sistemas se presenta en la Figura 2. 
Tabla 5. Velocidades de reacción calculadas para sistemas con solo crudo y crudo - agua a $8,27 \mathrm{MPa}$ (1200 psi).

\begin{tabular}{|c|c|c|c|}
\hline Sistema & $\begin{array}{l}\text { Temperatura } \\
\text { Promedio }\left[{ }^{\circ} \mathrm{C}\right]\end{array}$ & $\begin{array}{c}\text { Tiempo } \\
{[\mathrm{s}]}\end{array}$ & $\begin{array}{c}\mathrm{K}[\mathrm{g} O 2 / \mathrm{g} \\
\left.\text { oil }^{*} \mathrm{~s}\right]\end{array}$ \\
\hline \multirow{3}{*}{ Crudo } & 101,19 & 86940 & 1,37 E-06 \\
\hline & 115,44 & 97380 & 2,91 E-06 \\
\hline & 133,33 & 89640 & 4,61 E-06 \\
\hline \multirow{3}{*}{$\begin{array}{l}\text { Crudo - } \\
\text { agua }\end{array}$} & 99,75 & 86640 & 9,06 E-07 \\
\hline & 119,92 & 86760 & 2,75 E-06 \\
\hline & 140,06 & 86760 & 4,18 E-06 \\
\hline
\end{tabular}

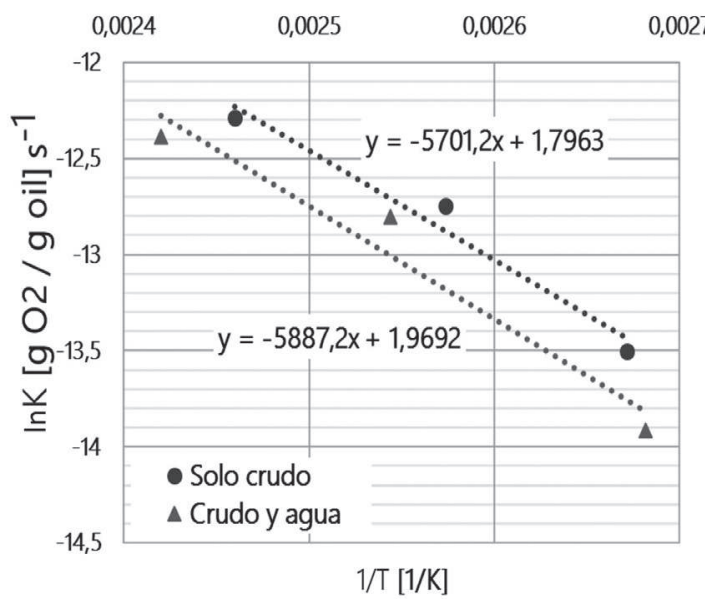

Figura 2. Cinética de reacción para sistemas con solo crudo y crudo - agua a $8,27 \mathrm{MPa}$ (1200 psi).
Los parámetros cinéticos correspondientes a cada sistema son presentados en la Tabla 6 .

Tabla 6. Parámetros cinéticos del sistema aceite - roca.

\begin{tabular}{ccc}
\hline & Parámetro & Valor \\
\hline \multirow{2}{*}{ Crudo } & $E / R[\mathrm{~K}]$ & $-5701,20$ \\
& $k_{\mathrm{o}}\left[1 / \mathrm{s}^{*} \mathrm{~atm}^{\mathrm{n}}\right]$ & 0,34 \\
& $E / R[\mathrm{~K}]$ & $-5887,20$ \\
Crudo - Agua & $k_{\mathrm{o}}\left[1 / \mathrm{s}^{*} \mathrm{~atm}^{\mathrm{n}}\right]$ & 0,41 \\
\hline
\end{tabular}

A partir de estas pruebas se logró determinar que no existe un efecto significativo del agua sobre la cinética de la reacción. Lo anterior debido a que los valores de energía de activación y de factor de frecuencia tuvieron poca variación para ambos sistemas. Las menores velocidades de reacción obtenidas en presencia de agua indica que la ignición será alcanzada en un mayor tiempo que en ausencia de agua.

\section{Simulación numérica de la ignición en un proceso de combustión in situ}

La simulación de la etapa de ignición de un proceso de CIS resulta conveniente para investigar la influencia de los parámetros operacionales y reflejar la respuesta dinámica del sistema (Figura 3).

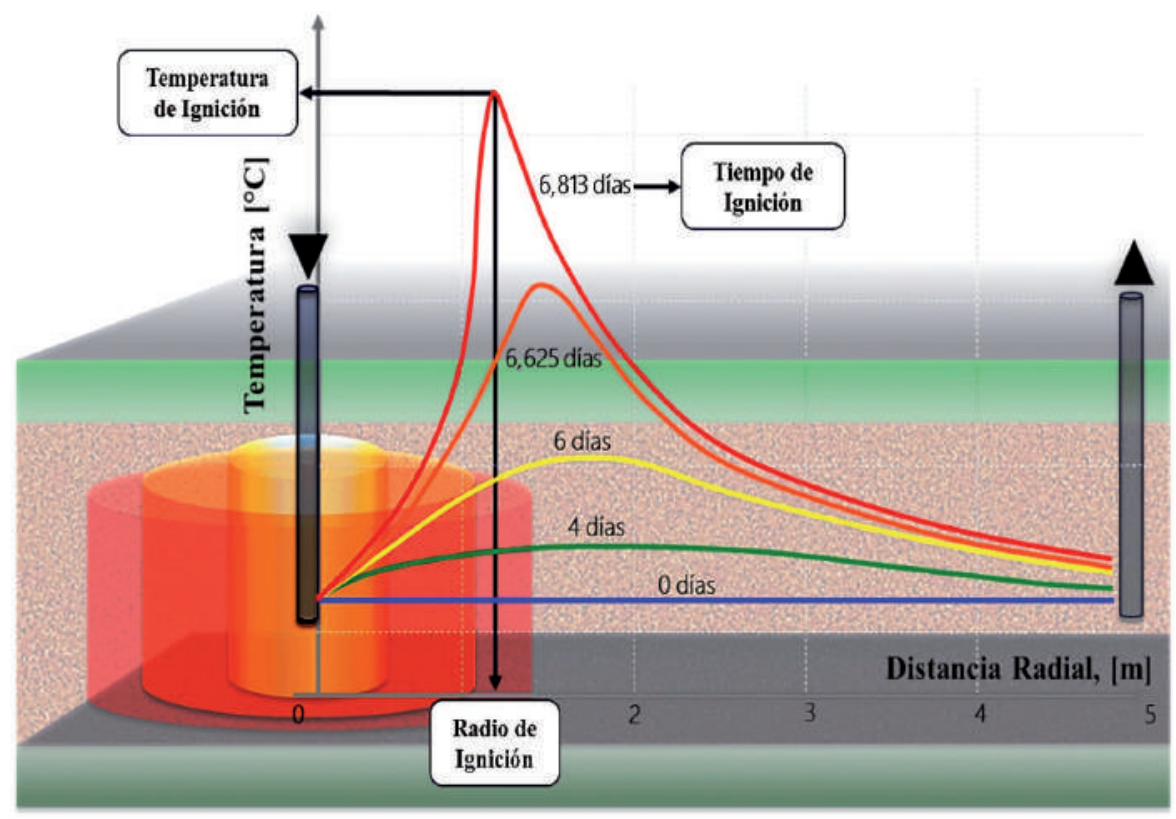

Figura 3. Comportamiento de la etapa de ignición en el proceso CIS. 


\section{Descripción del modelo diferencial}

Schoeppel y Ersoy [10] propusieron un modelo 2D cilíndrico con un patrón de cinco puntos invertido, en el cual el aire será inyectado en una sección horizontal y homogénea del yacimiento. El modelo matemático asume que la presión del sistema es constante, la concentración de oxígeno y la temperatura tendrán variaciones insignificantes a lo largo del eje vertical, la transferencia de calor se asume por conducción y por convección y el consumo de oxígeno es compensado por el proceso de difusión y la inyección continua de aire. Las ecuaciones que gobiernan el proceso de ignición espontánea son:

Balance de energía (Ecuación 7): Se tiene en cuenta,
-Ley Fourier. Transferencia de calor por conducción. -Convección dentro de la fase gas.

-Ley Arrhenius. Calor liberado mediante la reacción.

-Calor acumulado dentro del sistema.

$$
\frac{\partial^{2} T}{\partial r^{2}}+\boldsymbol{\alpha}_{e} \frac{1}{r} \frac{\partial T}{\partial r}+\boldsymbol{\beta}_{e} C^{n} e^{-E / R T}=\gamma_{e} \frac{\partial T}{\partial t}
$$

Donde,

$$
\boldsymbol{\alpha}_{\boldsymbol{e}}=1-\frac{\left(\rho c_{p} w\right)}{2 \pi k} ; \gamma_{\boldsymbol{e}}=\frac{(\rho C)^{*}}{k} ; \boldsymbol{\beta}_{\boldsymbol{e}}=\frac{\emptyset \rho_{o} S_{o} K_{o} Q_{m o l} R^{n} T_{i n y}^{n} X_{O 2}^{n}}{k^{*} M_{o 2}}
$$

La capacidad calorífica equivalente por unidad de volumen poroso se define como,

$$
(\rho C)^{*}=(1-\varnothing)(\rho C)_{\text {matriz }}+\varnothing\left[(\rho C)_{o} S_{o}+(\rho C)_{w} S_{w}+(\rho C)_{g} S_{g}\right]
$$

Balance de masa (Ecuación 9): Se tiene en -Transferencia de oxígeno por convección. cuenta, -Transferencia de oxígeno por difusión.

-Oxígeno consumido por la reacción química. -Oxígeno acumulado dentro del sistema.

$$
\frac{\partial^{2} C}{\partial r^{2}}+\boldsymbol{\alpha}_{\boldsymbol{m}} \frac{1}{r} \frac{\partial C}{\partial r}-\boldsymbol{\beta}_{\boldsymbol{m}} C^{n} e^{-E /} R T=\gamma_{m} \frac{\partial C}{\partial t}
$$

Donde,

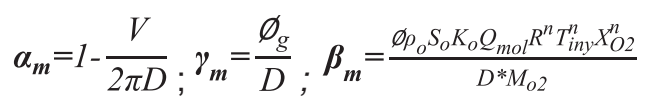

El sistema de ecuaciones diferenciales parciales acopladas por la ecuación de Arrhenius fue discretizada por diferencias finitas, y solucionado mediante el método numérico de Crank Nicolson. El modelo matemático fue programado en el programa MATLAB, el cual permite al usuario ingresar los parámetros de yacimiento, parámetros operacionales y parámetros cinéticos, así como también permite definir las dimensiones en espacio y tiempo. Los datos presentados a continuación fueron suministrados por el ICP (Tabla 7).

Condiciones Iniciales y de Frontera del modelo.

\begin{tabular}{cc}
\hline \multicolumn{2}{c}{ Condiciones Iniciales } \\
\hline$T(r, t=0)=T R$ & $X O_{2}(r, t=0)=0,21$ \\
\hline \multicolumn{2}{c}{ Condiciones de Frontera } \\
\hline En el pozo & Límite de la zona \\
investigada \\
$T(r=r w, t)=T w$ & $T(r=r e, t)=T R$ \\
$X O_{2}(r=r w, t)=0,21$ & $X O_{2}(r=r e, t)=0,21$ \\
\hline
\end{tabular}

$T R=$ Temperatura del yacimiento $T w=$ Temperatura del pozo inyector 
Tabla 7. Datos de entrada para la simulación numérica de la ignición espontánea.

\begin{tabular}{|c|c|c|}
\hline \multicolumn{3}{|c|}{ Propiedades térmicas del sistema modelo } \\
\hline Parámetro & Valor & Unidades \\
\hline Conductividad térmica del aceite & $3,35 \mathrm{E}-07$ & $\mathrm{kcal} / \mathrm{cm}^{*} \mathrm{~s}^{* \circ} \mathrm{C}$ \\
\hline Conductividad térmica del agua & $1,14 \mathrm{E}-06$ & $\mathrm{kcal} / \mathrm{cm}^{*} \mathrm{~s}^{* \circ} \mathrm{C}$ \\
\hline Conductividad térmica del gas & $1,12 \mathrm{E}-07$ & $\mathrm{kcal} / \mathrm{cm}^{*} \mathrm{~s}^{* \circ} \mathrm{C}$ \\
\hline Conductividad térmica de la arena & 2,00 E-05 & $\mathrm{kcal} / \mathrm{cm}^{*} \mathrm{~s}^{* \circ} \mathrm{C}$ \\
\hline Calor específico del aceite & 2,84 E-04 & $\mathrm{kcal} / \mathrm{g}^{* \circ} \mathrm{C}$ \\
\hline Calor específico del agua & $5,56 \mathrm{E}-04$ & $\mathrm{kcal} / \mathrm{g}^{* \circ} \mathrm{C}$ \\
\hline Calor específico del gas & $1,35 \mathrm{E}-04$ & $\mathrm{kcal} / \mathrm{g}^{* \circ} \mathrm{C}$ \\
\hline Calor específico de la arena & $1,04 \mathrm{E}-04$ & $\mathrm{kcal} / \mathrm{g}^{* \circ} \mathrm{C}$ \\
\hline \multicolumn{3}{|c|}{ Propiedades de los fluidos del sistema modelo } \\
\hline Parámetro & Valor & Unidades \\
\hline Densidad del aceite & 1,01 & $\mathrm{~g} / \mathrm{cm}^{3}$ \\
\hline Densidad del agua & 1,00 & $\mathrm{~g} / \mathrm{cm}^{3}$ \\
\hline Densidad del gas & $1,20 \mathrm{E}-03$ & $\mathrm{~g} / \mathrm{cm}^{3}$ \\
\hline Densidad de la arena & 2,65 & $\mathrm{~g} / \mathrm{cm}^{3}$ \\
\hline \multicolumn{3}{|c|}{ Datos físico-químicos del sistema modelo } \\
\hline Parámetro & Valor & Unidades \\
\hline Calor Liberado & 95 & $\mathrm{kcal} / \mathrm{mol} \mathrm{O}_{2}$ \\
\hline Fracción molar de oxígeno & 0,21 & - \\
\hline Coeficiente de difusión & 0,18 & $\mathrm{~cm}^{2} / \mathrm{s}$ \\
\hline Presión del sistema & 8,27 & $\mathrm{MPa}$ \\
\hline Temperatura del yacimiento & 86 & ${ }^{\circ} \mathrm{C}$ \\
\hline Porosidad & 0,17 & - \\
\hline Saturación de aceite & 0,55 & - \\
\hline Saturación de agua & 0,33 & - \\
\hline Saturación de gas & 0,12 & - \\
\hline \multicolumn{3}{|c|}{ Parámetros cinéticos del sistema roca fluido a $8.27 \mathrm{MPa}$ (1200 psi) para un $n=1$} \\
\hline Parámetro & Valor & Unidades \\
\hline Termino de activación & 5600 & $\mathrm{~K}$ \\
\hline Orden de reacción & 1 & - \\
\hline Factor pre-exponencial & 0,27 & $1 / \mathrm{s}^{*} \mathrm{~atm}^{\mathrm{n}}$ \\
\hline \multicolumn{3}{|c|}{ Condiciones de inyección para sistema modelo } \\
\hline Parámetro & Valor & Unidades \\
\hline Temperatura del pozo & 86 & ${ }^{\circ} \mathrm{C}$ \\
\hline Temperatura de inyección & 86 & ${ }^{\circ} \mathrm{C}$ \\
\hline Tasa de inyección de gas & 115,74 & $\mathrm{cc} / \mathrm{s}^{*} \mathrm{~cm}$ \\
\hline Presión de inyección & 12,16 & $\mathrm{MPa}$ \\
\hline
\end{tabular}


Los pasos en espacio y tiempo empleados para el modelo fue de $25 \mathrm{~cm}$ y $300 \mathrm{~s}$ respectivamente. De esta manera no se excedió de 0,5 la relación del paso del tiempo con respecto al cuadrado del paso de la distancia, como se recomienda al aplicar el método numérico de Crank Nicolson.

\section{Validación del modelo matemático programa- do en MATLAB}

El efecto de diferentes parámetros operacionales sobre la ignición fue evaluado por Shoeppel y Ersoy en 1968 [10]. Su estudio mostró que la tasa de inyección tenía una influencia directa sobre el pico de temperatura alcanzada en la ignición y sobre la forma del perfil de temperatura. En el modelo programado para el presente estudio, se emplearon las tasas de inyección de 500, 1000 y 2000 m³/día/m (Figura 4). Se validó entonces el incremento en el pico de temperatura alcanzado y el desplazamiento del frente de combustión hacia el pozo productor en el caso de la mayor tasa de aire inyectada. Se tiene un comportamiento opuesto del perfil de temperatura con menores tasas de inyección, fenómeno que sucede en todas las implementanciones de $\mathrm{CIS}$, ya que resulta imposible mantener las mismas tasas de inyección en todo el sistema roca-fluido, debido principalmente a heterogeneidades. De acuerdo a la temperatura alcanzada se desarrollarán diferentes régimenes de reacción (LTO, IT, HTO).

La distancia a la cual toma lugar la ignición espontánea desde el pozo inyector fue reportada por Burger [12] en función de las tasas de inyección. El estudio indicó que si la ignición ocurre cerca del pozo, el tiempo de retardo incrementa ligeramente cuando la tasa de inyección disminuye. Para el modelo construido y con los mismos casos de tasas de inyección se calcularon los tiempos de retardo, cumpliendo con el comportamiento de Burger (Figura 5). Mayores pérdidas de calor cerca del pozo hacen que el tiempo de retardo sea mayor, mientras que las altas tasas de inyección promueven un rápido transporte de calor a cierta distancia del pozo inyector.

Finalmente, para terminar la validación del simulador, se hace referencia al efecto de la reactividad del crudo sobre la temperatura del sistema, el cuál fue reportado por Shoeppel y Ersoy [10]. En su estudio, la energía de activación fue uno de los parámetros que más impactaba la temperatura alcanzada después de determinado tiempo, comportamiento que se evidenció en nuestro modelo variando el termino de activación E/R desde $5600 \mathrm{~K}$ hasta $6000 \mathrm{~K}$ (Figura 6). Pequeños cambios en el valor del término de activación del sistema pueden demandar mayores tasas de calentamiento, y por ende tomará mucho más tiempo en alcanzar la ignición espontánea, lo que significa que se deben evaluar métodos de ignición asistida que cambién las propiedes químicas del medio.

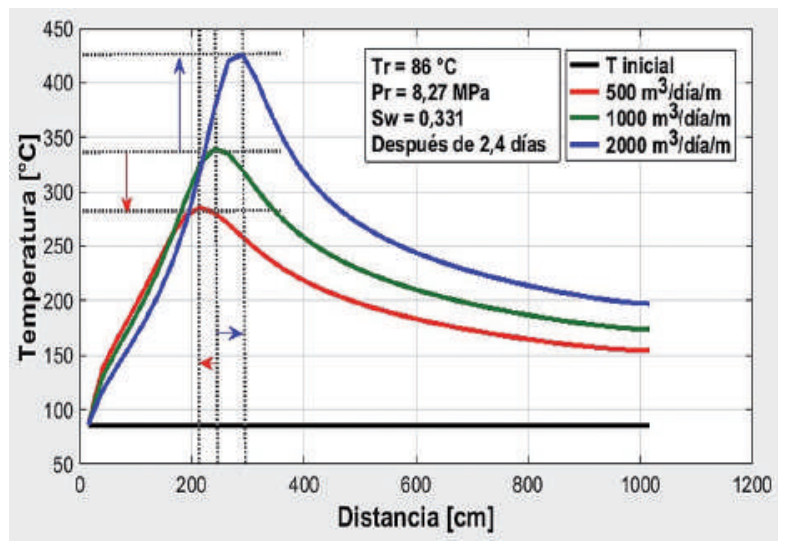

Figura 4. Efecto de la tasa de inyección sobre la temperatura.

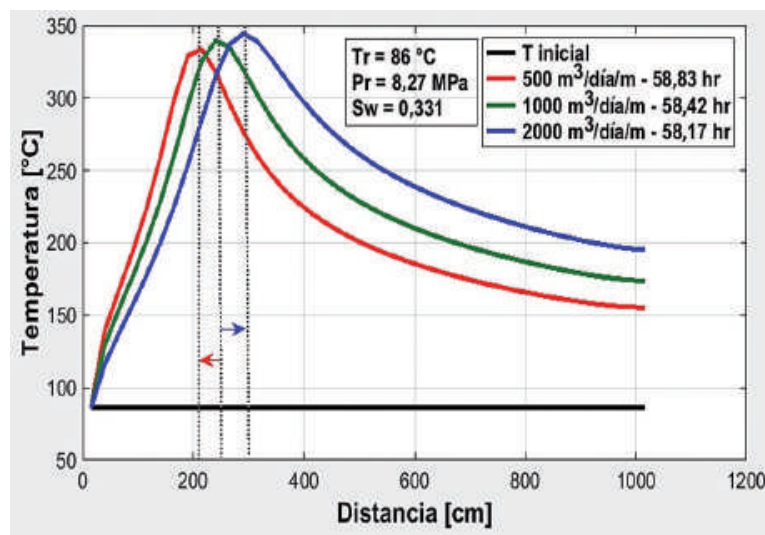

Figura 5. Efecto de la tasa de inyección sobre el retardo y radio de ignición.

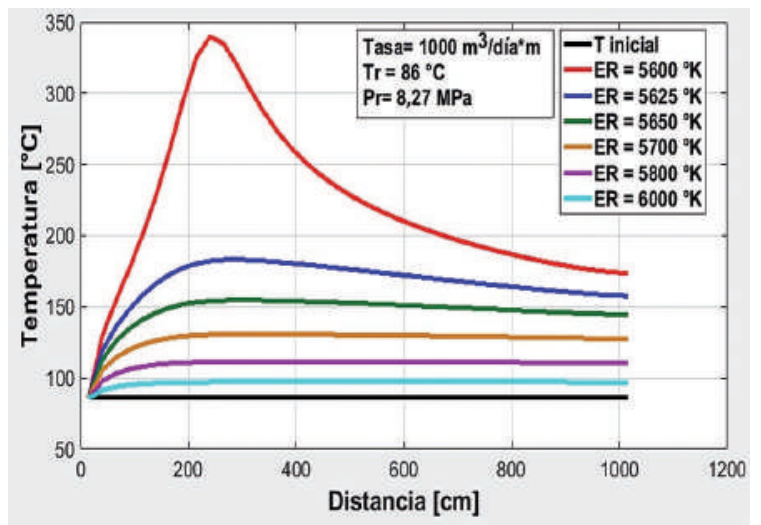

Figura 6. Efecto de la energía de activación sobre la temperatura. 
Predicción de la etapa de ignición para un yacimiento de crudo pesado

Considerando las condiciones actuales de presión y temperatura del campo modelo en estudio e ingresando los valores de las propiedades del sistema roca-fluido y los parámetros cinéticos, se obtuvieron los perfiles de temperatura y concentración de oxígeno en función de la distancia y del tiempo (Figuras 7 -10).

En los perfiles se observa cómo la temperatura del yacimiento empieza a incrementar debido a que las tasas de liberación de energía por las reacciones de oxidación superan las tasas de disipación de energía en el medio (Figura 7). El consumo de oxígeno inicia desde que el aire hace contacto con el hidrocarburo, mediante las reacciones de oxidación a bajas temperaturas $\left(50^{\circ} \mathrm{C}-350^{\circ} \mathrm{C}\right)$, en los cuales se espera la formación de hidrocarburos oxigenados, dióxido de carbono, monóxido de carbono y agua. Cuando se alcanza la temperatura de ignición, la mayor parte del oxígeno es consumido, como se observa en la Figura 9. La temperatura de ignición alcanzada fue de $340{ }^{\circ} \mathrm{C}$, la cual fue suministrada por el laboratorio de inyección de aire del ICP, valor obtenido a partir de una prueba RTO (Ramped temperature oxidation). Las reacciones de combustión desencadenadas por la ignición espontánea liberan grandes cantidades de energía que se va desplazando frontalmente hacia el pozo productor, en la dirección del flujo de aire. Debido al flujo radial, un mayor volumen de roca con capacidad calorífica es contactado por el aire inyectado. Por lo tanto, el calor es absorbido por el sistema roca-fluido lo que conduce a que la temperatura disminuya después del punto de ignición.

De los perfiles se puede inferir que el tiempo que tarda el sistema para alcanzar la temperatura de ignición, también conocido como tiempo de retardo, es ligeramente inferior a 58,4 horas $(2,43$ días). Adicionalmente, la ignición espontánea tuvo lugar aproximadamente a 2.404 metros del pozo inyector.

Estos resultados son análogos a las predicciones realizadas por Rao et al [15], para el campo Balol en India. Un yacimiento de características similares al estudiado en esta investigación, con una temperatura de $70^{\circ} \mathrm{C}$. La temperatura de ignición estimada en pruebas de laboratorio fue de $200^{\circ} \mathrm{C}$, lo que indicaba una alta reactividad del crudo. Las predicciones establecieron que la ignición se daría entre 1,46 días a 2,72 días, a 1,524m de distancia desde el pozo inyector.

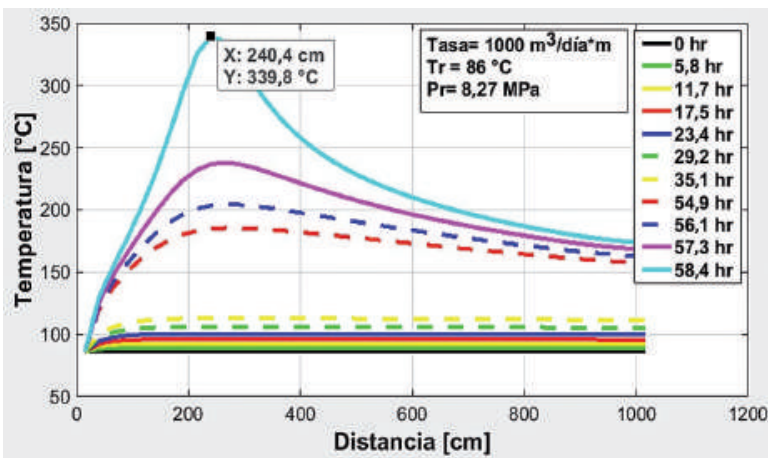

Figura 7. Perfiles de temperatura Vs. distancia.

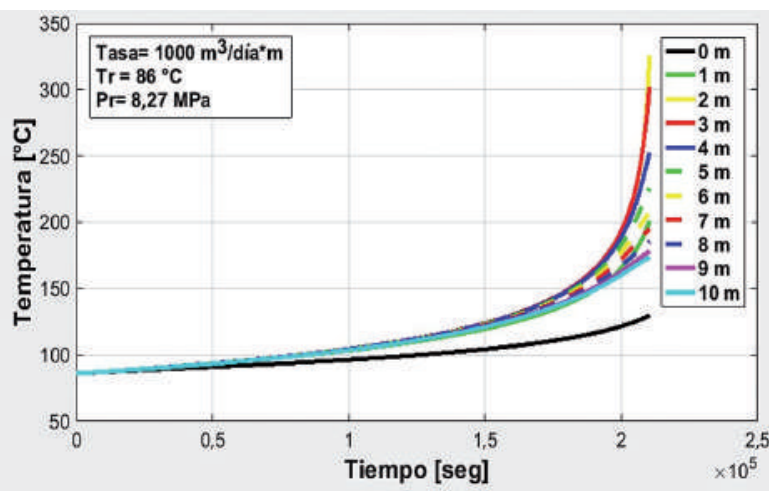

Figura 8. Perfiles de temperatura Vs. tiempo .

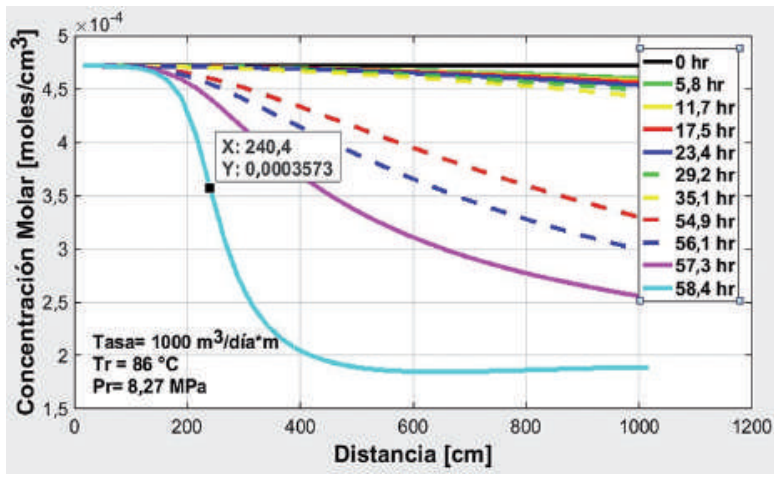

Figura 9. Perfiles de concentración de $\mathrm{O}_{2}$ Vs. distancia.

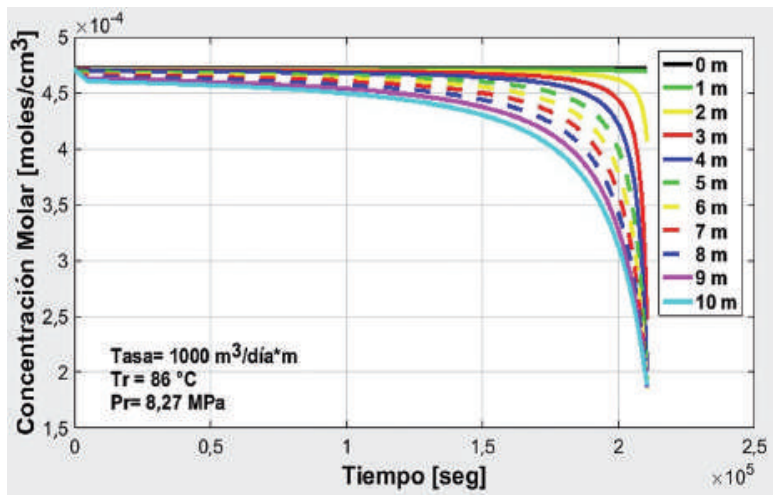

Figura 10. Perfiles de concentración de $\mathrm{O}_{2}$ Vs. tiempo. 


\section{Efecto de la saturación de agua sobre la etapa de ignición}

Los yacimientos de crudo pesado son generalmente encontrados con agua de formación, agua que ha sido inyectada en procesos de recuperación secundaria o agua como vapor condensado luego de una recuperación terciaria. En zonas con altas saturaciones de aceite se pueden encontrar saturaciones de agua entre el $10 \%$ y el $30 \%$ [16]. Lo anterior implica que la oxidación del hidrocarburo en una aplicación del proceso de CIS ocurrirá en presencia del agua.

Para evaluar el efecto de la saturación de agua (Sw) sobre la etapa de ignición de un proceso CIS se establece un rango de Sw entre 33,1\% - 70\%, donde el límite inferior corresponde al yacimiento considerado para este estudio, mientras que el límite superior se encuentra en función del mínimo contenido de aceite que permita compensar el consumo de aceite como combustible, cubrir los requerimientos de energía del proceso y suministrar una producción adicional para hacer el proceso rentable [17].

Las distribuciones de temperatura y de la concentración de oxígeno desde el pozo inyector hasta el pozo productor para cada uno de los escenarios de saturación de agua son presentados en la Figura 11 y Figura 12 respectivamente. La Figura 13 presenta el tiempo de retardo para cada uno de los escenarios de saturación de agua. Todos los escenarios fueron estudiados con una tasa de inyección constante de $1000 \mathrm{~m}^{3} / \mathrm{día} / \mathrm{m}$, y manteniendo las mismas propiedades físicoquímicas del modelo base. Los perfiles muestran que todos los casos alcanzaron la temperatura de ignición espontánea en distintos tiempos de retardo y distintas posiciones desde el pozo inyector.

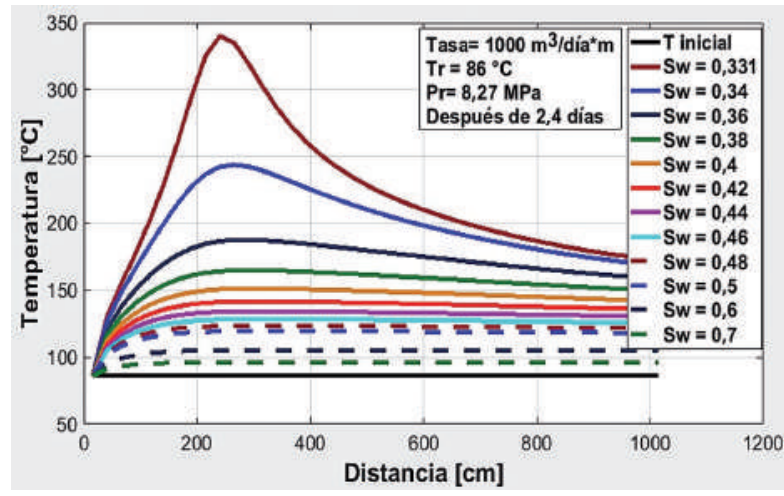

Figura 11. Efecto de la saturación de agua sobre la temperatura.

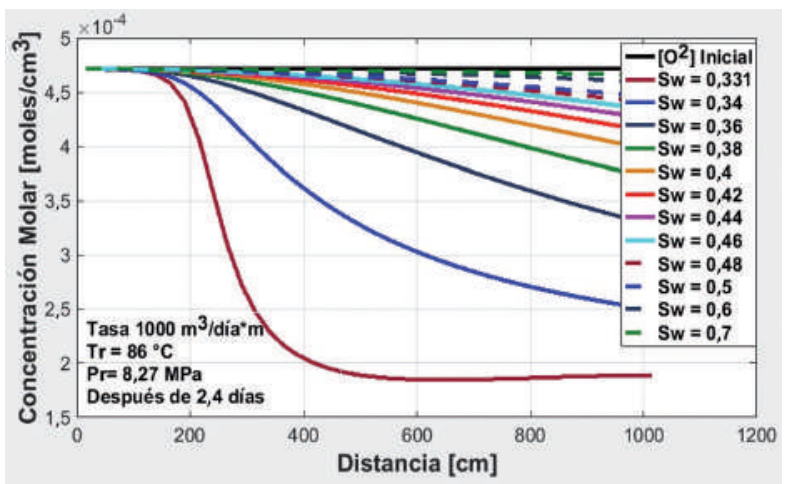

Figura 12. Efecto de la saturación de agua sobre la concentración de $\mathrm{O}_{2}$.

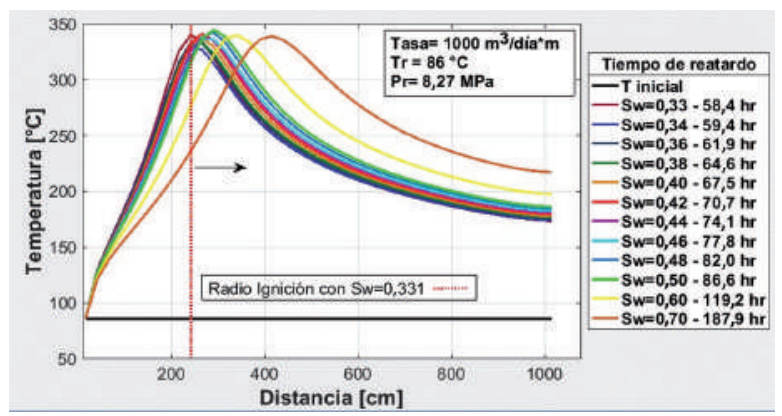

Figura 13. Efecto de la saturación de agua sobre el retardo y radio de la ignición espontánea.

\section{Discusión de resultados}

Diversas investigaciones se han concentrado en destacar el papel que desempeña la presencia de agua en el estado estable de un proceso CIS, a tal punto que se evalúan exitosamente modalidades de combustión húmeda, esto atribuido principalmente a que la presencia de agua crea un ambiente adecuado para altas tasas de calentamiento, donde el calor latente y la interacción del vapor con los hidrocarburos mejoran las características oxidativas del crudo en sitio. Adicionalmente, los productos oxigenados formados durante reacciones de oxidación parcial (LTO) pueden someterse a reacciones subsecuentes, liberando grandes cantidades de energía, manteniendo la sostenibilidad del proceso de combustión en todo el yacimiento [9].

El efecto de la saturación de agua sobre la temperatura alcanzada a un tiempo de 2,4 días puede observarse en la Figura 11. Los perfiles permiten afirmar que en sistemas con mayores saturaciones de agua la tasa de liberación de energía por las reacciones de oxidación no es lo 
suficientemente alta para elevar la temperatura del sistema, ya que una mayor parte del calor liberado es acumulado en el sistema. La razón para dicha acumulación incremental es el aumento en la capacidad calorífica equivalente del sistema, en el que se incluye la arena del yacimiento, el aceite, el gas y el agua, siendo este último el que posee mayor calor específico de los tres fluidos presentes en distintas proporciones en el yacimiento. De esta manera, al incrementar el contenido de agua, el sistema requiere más energía térmica para elevar la temperatura.

Para evaluar el efecto de la saturación de agua sobre el tiempo de retardo, se determinó numéricamente el tiempo que tardó cada sistema en elevar la temperatura hasta el punto de ignición $\left(340^{\circ} \mathrm{C}\right.$, prueba RTO). Por otro lado, se consideró calcular los tiempos de ignición para los mismos sistemas de forma analítica, haciendo uso de la correlación de Tadema y Weijdema [11], el cual simplifica el cálculo del tiempo de ignición omitiendo los efectos de transferencia de calor por conducción y convección (Ecuación 10).

$$
T_{\text {ignición }}=(\rho c)^{*} \frac{M_{O 2} T_{R}^{2}\left(1+\frac{2 R T_{R}}{E}\right)}{\emptyset_{o} S_{o} Q k_{o} P_{O 2}^{n} \frac{E}{R}} e^{\frac{E}{R T_{R}}}
$$

Donde,

$(\rho c)^{*}=(1-\varnothing)(\rho C)_{\text {matriz }}+\varnothing\left[(\rho C)_{o} S_{o}+(\rho C)_{w} S_{w}+(\rho C)_{g} S_{g}\right]$

La Figura 14 permite hacer la comparación entre los tiempos de ignición determinadas por la ecuación analítica con los tiempos observados en los perfiles de temperatura y oxígeno obtenidos por el modelo matemático programado. Los tiempos de retardo obtenidos por ambos métodos difieren en más del $100 \%$ de sus magnitudes, señalando la importancia de considerar los efectos de transferencia de calor por convección y conducción al momento de evaluar la etapa de ignición, ya que se puede sobrestimar la reactividad de un sistema roca-fluido en condiciones específicas. Burger [12], también identificó que el método numérico aproximaba mejor el cálculo del tiempo de ignición [12].

Por otro lado, el tiempo de ignición se relaciona exponencialmente con el incremento de la saturación de agua, debido a la mayor cantidad de energía térmica requerida para elevar la temperatura de un mayor volumen del yacimiento contactado a medida que el aire es inyectado. Esto es soportado por considerar flujo radial en el modelo matemático programado.

La Figura 14 también puede ser empleada para analizar el efecto la tasa de inyección sobre el tiempo de ignición en función de la saturación de agua. Las tasas que se utilizaron fueron 200 , 500, 1000 (Modelo base), 1500 y 3000 m²/día/m. Resulta evidente la poca influencia que tiene la tasa de inyección sobre el tiempo para alcanzar la ignición espontánea. Sin embargo, se nota una ligera tendencia a disminuir el tiempo de retardo cuando se incrementa la tasa de inyección, debido a que se dispone de una mayor capacidad para desplazar más eficientemente el frente de calor desde el pozo inyector en un menor tiempo.

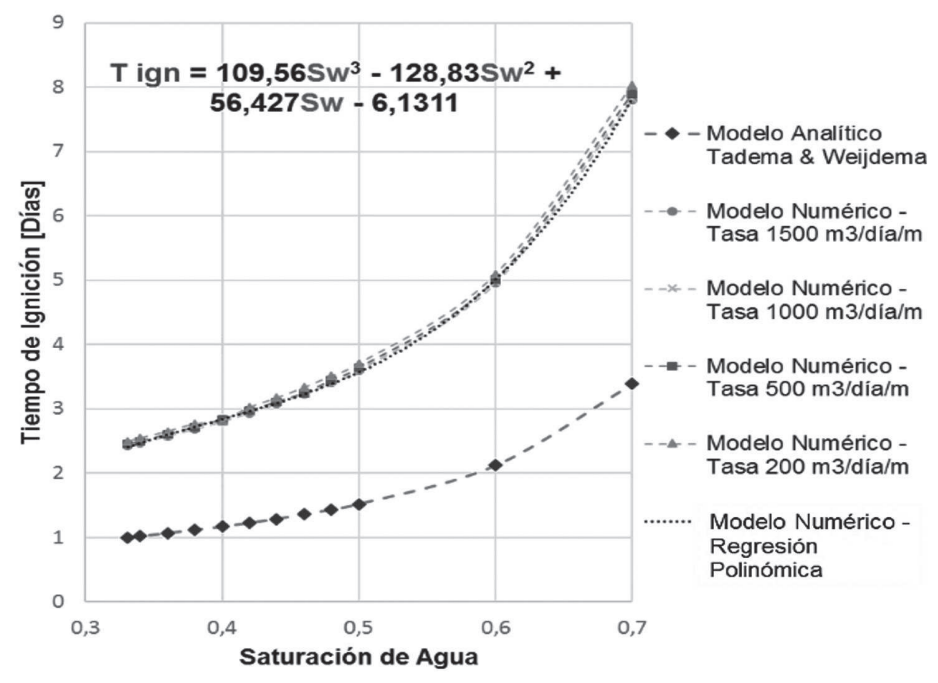

Figura 14. Comparación del tiempo de ignición espontánea Método numérico Vs Método analítico. 
Lo anterior indica que altas inversiones en proporcionar mayores tasas de inyección para reducir el tiempo de ignición resulta innecesaria, porque no se obtendrán grandes efectos. Pero si la intención es alejar el inicio de la ignición de la cara de pozo por términos de seguridad, la tasa de inyección de aire si puede otorgar el resultado esperado. Lo anterior se puede observar en la Figura 15, afirmando que mayores tasas de inyección promueven que la ignición tome lugar a una mayor distancia del pozo inyector en aproximadamente el mismo tiempo, como se mencionó previamente.

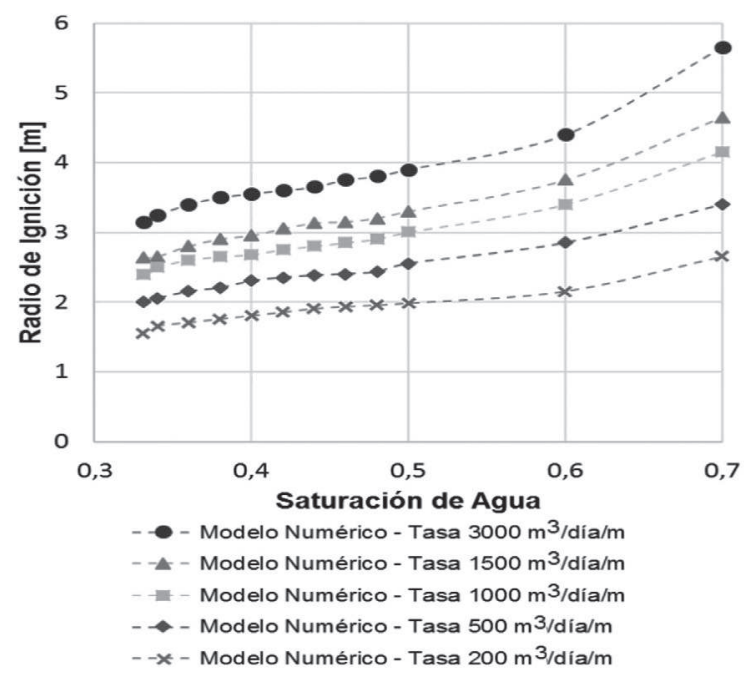

Figura 15. Efecto de la saturación de agua sobre la distancia a la cual ocurre la ignición espontánea.

El radio de ignición incrementa con la saturación de agua, como se pudo observar en la Figura 13. De la Figura 15 se puede destacar el efecto que tiene en conjunto incrementar la saturación de agua y la tasa de inyección para obtener un radio seguro de ignición espontánea en un proceso de CIS, lo que permitirá garantizar la integridad del completamiento del pozo inyector y la protección de la vida de los operarios en campo. Lo anterior indica que si para el caso base (tasa de inyección $1000 \mathrm{~m}^{3} / \mathrm{día} / \mathrm{m}$ y una $\mathrm{Sw}$ de 0,331 ) se requiere un radio de ignición por encima de los $3 \mathrm{~m}$, se puede optar por incrementar la tasa de inyección a $3000 \mathrm{~m}^{3} / \mathrm{día} / \mathrm{m}$ o elevar la saturación de agua en las cercanías del pozo inyector a 0,5 (Figura 15). Una saturación de agua de 0,5 requerirá un mayor tiempo de retardo, aproximadamente 1 día más del caso base inyectando la misma tasa constante de
$1000 \mathrm{~m}^{3} / \mathrm{día} / \mathrm{m}$, reduciendo de esta manera costos de compresión.

Este análisis debe ser considerado junto con el mejoramiento en el comportamiento oxidativo del crudo en presencia de agua y la obtención de productos LTO con menor efecto destructivo sobre la sostenibilidad del proceso CIS. Sin embargo, debe primarse la presencia de altas saturaciones de aceite alrededor del pozo inyector que permitan un ambiente con altas tasas de calentamiento para lograr la ignición espontánea y un subsecuente proceso de CIS sostenido.

\section{Conclusiones}

El efecto de la saturación de agua sobre la ignición fue evaluado para sistemas con diferentes contenidos agua, mostrando efectos directos sobre el tiempo de retardo y la distancia a la cual se genera la ignición desde el pozo inyector. Debido a que la presencia de agua aumenta la capacidad calorífica equivalente del sistema, haciendo que mayor parte del calor generado por las reacciones de oxidación sea absorbido por el sistema, impidiendo el aumento de la temperatura en el yacimiento.

La cinética de oxidación de dos muestras, una de crudo y otra de crudo con agua fue estudiada mediante la celda de oxidación isotérmica del ICP bajo condiciones cercanas a las de yacimiento. Los parámetros cinéticos obtenidos para ambas muestras no presentaron variaciones significativas, indicando que el agua no afecta directamente la cinética de reacción, en cambio ésta sólo dependerá de la composición química del crudo, la concentración de los reactantes, la temperatura y presencia de catalizadores en el sistema roca-fluido.

Estudios de laboratorio confirman la importancia del agua para lograr la sostenibilidad de un proceso de combustión in situ en estado estable. La producción de cetonas en medio acuoso permite liberar mayores cantidades de energía, así como también se obtendrá menor formación de coque, la casi eliminación del ácido sulfhídrico y productos LTO con menor viscosidad y acidez en presencia de agua, que crean un ambiente sostenible para altas tasas de calentamiento y con esto el avance estable del frente de combustión en régimen HTO. 


\section{Agradecimientos}

Los autores agradecen al Instituto Colombiano del Petróleo por suministrar la información de las pruebas de laboratorio, al soporte técnico del Grupo de Investigación de Recobro Mejorado, GRM, y al Ing. Humberto Lizcano por sus valiosos aportes.

\section{Referencias bibliográficas}

[1] Larter S. The origin, prediction and impact of oil viscosity heterogeneity on the production characteristics of tar sand and heavy oil reservoirs. J. Can. Petrol. Technol. 2008;47:52-61.

[2] Xia T, Greaves M, Turta A, Ayasse C. THAI - A 'short-distance displacement' in situ combustion process for the recovery upgrading of heavy oil. Chem. Eng. Res. Des. 2003;81:295-304.

[3] Instituto Colombiano del Petróleo, ECOPETROL S.A. Determinación del mecanismo de ignición, del tiempo de retardo y de los efectos del aceite de linaza para la inyección de aire en el campo Chichimene. Piedecuesta, Colombia: Lizcano H; 2015.

[4] Bilijderveen M. Ignition and combustion phenomena on a movin grate (tesis doctoral). Enschede, Netherlands: University of Twente; 2011.

[5] Turta A. In Situ Combustion. En: Enhanced Oil Recovery: Field cases studies. London: Gulf Professional Publishing; 2013. p. 447 - 541. ISBN-13: 978-0123865458.

[6] Burger J, Sahuquet B. Chemical aspects of in situ combustion-heat of combustion and kinetics. Soc. Pet. Eng. Journal. 1972;5(12):410-22.

[7] Khansari Z. A new reaction model for low temperature oxidation of heavy oil: Experiments and numerical modeling. Energy Elsevier. 2014;64:419-28.

[8] Lee D, Noureldin N. Effect of Water on the Low - Temperature Oxidation of Heavy Oil. Energy \& Fuels. 1989;3:713-5.

[9] Klock K, Hascakir B. Simplified Reaction Kinetics Model for In-Situ Combustion. Society of Petroleum Engineers; 2015. SPE-177134-MS.

[10] Schoeppel R, Ersoy D. Prediction of Spontaneous Ignition in In-Situ Combustion. Society of Petroleum Engineers; 1968. SPE 2383.

[11] Tadema H, Wijedma J. Spontaneous Ignition of Oil Sands. The Oil and Gas Journal. 1970;68(50):77-80.

[12] Burger J. Spontaneous Ignition in Oil Reservoirs.
Society of Petroleum Engineers Journal. 1976;16(2):73-81.

[13] Agca C, Yortsos YC. Steady-State Analysis of In-Situ Combustion. United States: Society of Petroleum Engineers; 1985.

[14] Aguillon M. Análisis de la etapa de ignición en la combustión in situ de crudo pesado (tesis pregrado). Medellin, Colombia: Universidad Nacional de Colombia; 2016.

[15] Rao N, Roychaudhury S, Sapkal A, Gupta K, Sinha S. Results Of Spontaneous Ignition Test in Balol Heavy Oil Field. Society of Petroleum Engineers; 1997. SPE-38067. p.435 - 441.

[16] Khansari Z. Low Temperature Oxidation of Heavy Crude Oil: Experimental Study and Reaction Modeling (tesis doctoral). Calgary, Canada: Department of Chemical and Petroleum Engineering-University of Calgary;2014

[17] Grupo de Investigación Recobro Mejorado, Universidad Industrial de Santander. Estudio de Combustión In Situ utilizando configuraciones horizontales en yacimiento: ventajas $y$ desventajas. Bucaramanga, Colombia: Dangon J, Muñoz S, Rojas J; 2016.

\section{Nomenclatura}

$\boldsymbol{k}_{\boldsymbol{o}}=$ Factor de Frecuencia, $\left[\mathrm{s}^{\star} \operatorname{atm}^{\mathrm{n}}\right]^{\wedge}(-1)$

$\boldsymbol{\rho}_{g}=$ Densidad del gas, $\left[\mathrm{g} / \mathrm{cm}^{3}\right]$;

$w=$ Tasa de inyección de aire, $\left[\mathrm{cm}^{3} / \mathrm{s}\right]$;

$\boldsymbol{k}=$ Conductividad térmica, $\left[\mathrm{kcal} / \mathrm{cm}^{*} \mathrm{~s}^{* \circ} \mathrm{C}\right.$;

$r=$ Radio, $[\mathrm{cm}]$

$\boldsymbol{K}=$ Velocidad de reacción, $\left[\mathrm{g}_{\mathrm{O} 2} / \mathrm{g}_{\circ}{ }^{*} \mathrm{~s}\right]$;

$C=$ Concentración de oxígeno, $\left[\mathrm{mol}_{\mathrm{O}_{2}} / \mathrm{cm}^{3}\right]$;

$-\boldsymbol{E} / \boldsymbol{R}=$ Termino de activación, [K];

$(\rho \mathrm{C})^{*}=$ Capacidad calorífica equivalente, $\left[\mathrm{kcal} / \mathrm{cm}^{3 * \circ} \mathrm{C}\right]$;

$\emptyset_{g}=$ Porosidad;

$\rho_{\boldsymbol{o}}=$ Densidad del aceite, $\left[\mathrm{g} / \mathrm{cm}^{3}\right]$;

$\boldsymbol{S}_{\boldsymbol{o}}=$ Saturación de aceite;

$S_{w}=$ Saturación de agua;

$\boldsymbol{S}_{g}=$ Saturación de gas;

$\boldsymbol{P}_{\mathrm{O}_{2}}=$ Presión parcial del oxígeno, [atm];

$n=$ Orden de reacción;

$\boldsymbol{M}_{\boldsymbol{O}_{2}}=$ Masa molar del oxígeno, $[\mathrm{g} / \mathrm{mol}]$;

$X_{O_{2}}=$ Fracción molar del oxígeno;

$T=$ Temperatura $\left[{ }^{\circ} \mathrm{C}\right]$;

$C_{o}=$ Calor especifico aceite, $\left[\mathrm{kcal} / \mathrm{g}^{* \circ} \mathrm{C}\right]$;

$C_{w}=$ Calor especifico agua, $\left[\mathrm{kcal} / \mathrm{g}^{* \circ} \mathrm{C}\right]$;

$C_{g}=$ Calor especifico gas, $\left[\mathrm{kcal} / \mathrm{g}^{* \circ} \mathrm{C}\right]$;

$\boldsymbol{D}=$ Coeficiente de difusión, $\left[\mathrm{cm}^{2} / \mathrm{s}\right]$;

$\boldsymbol{R}=$ Constante de los gases, [atm* $\left.{ }^{*} \mathrm{~cm}^{3} / \mathrm{K}^{*} \mathrm{~mol}\right] ;$

$T_{\text {iny }}=$ Temperatura de inyección, $\left[{ }^{\circ} \mathrm{C}\right]$;

$T_{w}=$ Temperatura en el pozo inyector, $\left[{ }^{\circ} \mathrm{C}\right]$;

$T R=$ Temperatura del yacimiento, $\left[{ }^{\circ} \mathrm{C}\right]$;

$r \boldsymbol{w}=$ Radio del pozo, $[\mathrm{cm}] ;$

$\boldsymbol{O}_{2 i}=$ Cantidad de oxígeno al inicio de la prueba, [mol]

$\boldsymbol{O}_{2 f}=$ Cantidad de oxígeno final, [mol];

$\boldsymbol{t}=$ Tiempo, [s];

$Z=$ Factor de comprensibilidad;

$M_{O}=$ Masa de aceite, [g]; 


Conversión de unidades
\begin{tabular}{l|l|l|l|l}
1 & $\mathrm{~cm}$ & $=$ & 0,001 & $\mathrm{~m}$ \\
1 & $\mathrm{~cm}^{3}$ & $=$ & $1,00 \mathrm{E}-06$ & $\mathrm{~m}^{3}$ \\
1 & $\mathrm{ft}$ & $=$ & 0,3048 & $\mathrm{~m}$ \\
1 & $\mathrm{~g}$ & $=$ & 0,001 & $\mathrm{~kg}$ \\
1 & $\mathrm{~g} / \mathrm{cm}^{3}$ & $=$ & 1000 & $\mathrm{~kg} / \mathrm{m}^{3}$ \\
1 & $\mathrm{~min}$ & $=$ & 60 & $\mathrm{~s}$ \\
1 & $\mathrm{Psi}$ & $=$ & $\mathrm{Pa}$
\end{tabular}

